ESH Abstract:

\title{
A COMPARISON OF THE IMPACT OF MORNING OR NIGHT DELIVERY OF ANTIHYPERTENSIVE AGENTS ON 24 HOUR AMBULATORY BLOOD PRESSURE MONITORING (ABPM) LEVELS: A RANDOMISED CROSS-OVER TRIAL \\ Objective
}

Some data suggest that nocturnal rather than daytime dosing of antihypertensive agents may have beneficial effects on consequent cardiovascular outcomes. This trial was designed to evaluate whether ABPM levels differ by timing of drug dosing as a possible explanation for these observations.

Design \& Method

103 male or female patients aged $18-80$ years with "controlled" hypertension $(\leq 150 / \leq 90 \mathrm{mmHg})$ for at least 3 months on stable therapy of $\geq 1$ antihypertensive agent, were recruited from 1 of 2 centres in London \& Thessaloniki. Patients were randomised to receive their usual therapy either in the morning $(6 \mathrm{am}-11 \mathrm{am})$ or the evening $(6 \mathrm{pm}-11 \mathrm{pm})$ for 12 weeks then participants crossed-over to the alternative timing of drug administration for a further 12 weeks.

Clinic BPs using standardised methodology and a 24 hour ABPM recording were taken at baseline 12 and 24 weeks as were routine blood tests and completion of the EQ-5D-5L questionnaire for quality of life. With 90 recruits completing the trial the study had $80 \%$ power $(\alpha=0.05)$ to detect a $3 \mathrm{mmHg}$ difference in mean $24 \mathrm{~h} \mathrm{SBP}$. Regression modelling was used to calculate confidence intervals adjusted by centre, period (visit), sequence (group).

Results

95 patients (92\%) completed all 3 ABPM recordings. The mean age of recruits was 52 years \& $44 \%$ were female.

24 hour systolic \& diastolic BPs did not differ between daytime \& nocturnal dosing (table).

Similarly daytime \& night time dosing had no differential impact on daytime ABPM levels (7.00 am$10.00 \mathrm{pm}) \&$ night time ABPM levels $(10.00 \mathrm{pm}-7.00 \mathrm{am})$ nor on clinic BP levels.

Stratification by age $(<65 / \geq 65$ years) or gender did not affect the results.

\begin{tabular}{|l|l|l|l|l|l|}
\hline $\begin{array}{c}\text { Outcome } \\
\text { (N=95) }\end{array}$ & \multicolumn{1}{|c|}{ Baseline } & \multicolumn{2}{|c|}{ Drug taken } & $\begin{array}{c}\text { Observed } \\
\text { difference }\end{array}$ & $\begin{array}{c}\text { Adjusted } \\
\text { Difference } \\
(95 \% \text { CI })\end{array}$ \\
\hline & & \multicolumn{1}{|c|}{ Morning } & \multicolumn{1}{|c|}{ Evening } & & \\
\hline 24h Systolic BP & 128.6 & 129.7 & 129.8 & 0.10 & $0.11(-3.20,3.42)$ \\
\hline 24h Diastolic BP & 76.9 & 77.2 & 78.0 & 0.75 & $0.77(-1.38,2.91)$ \\
\hline Day time SBP & 131.2 & 132.2 & 132.8 & 0.53 & $0.54(-2.82,3.89)$ \\
\hline Day time DBP & 79.1 & 79.3 & 80.6 & 1.28 & $1.30(-0.96,3.56)$ \\
\hline Night time SBP & 120.9 & 122.8 & 121.1 & -1.68 & $-1.62(-5.38,2.15)$ \\
\hline Night time DBP & 69.8 & 70.9 & 70.6 & -0.35 & $-0.32(-2.81,2.17)$ \\
\hline Clinic SBP & 128.1 & 129.4 & 129.8 & 0.44 & $0.39(-2.91,3.69)$ \\
\hline Clinic DBP & 76.5 & 77.3 & 77.4 & 0.15 & $0.14(-2.03,2.32)$ \\
\hline $\begin{array}{l}\text { Quality of Life } \\
\text { Score }\end{array}$ & 82.8 & 84.1 & 84.0 & -0.10 & $-0.12(-3.12,2.89)$ \\
\hline
\end{tabular}

Conclusions

In treated hypertensive patients with stable BP levels the timing of antihypertensive drug administration (morning or evening) did not affect mean 24 hour ABPM levels. 